\title{
Low triiodothyronine syndrome as a predictor of poor outcomes in patients undergoing brain tumor surgery: a pilot study
}

\author{
Clinical article
}

\author{
Adomas Bunevicius, M.D., Ph.D., ${ }^{1,3,4}$ Vytenis Deltuva, M.D., Ph.D., ${ }^{1,2}$ \\ Sarunas Tamasauskas, M.D., ${ }^{1}$ Arimantas Tamasauskas, M.D., Ph.D., ${ }^{1,2}$ \\ Ejward R. Laws JR., M.D., 5 and Robertas Bunevicius, M.D., Ph.D. ${ }^{3}$
}

${ }^{1}$ Department of Neurosurgery and ${ }^{2}$ Institute of Neurosciences, Lithuanian University of Health Sciences, Kaunas; ${ }^{3}$ Behavioral Medicine Institute, Lithuanian University of Health Sciences, Palanga, Lithuania; ${ }^{4}$ Department of Neurology, University of North Carolina at Chapel Hill, North Carolina; and ${ }^{5}$ Department of Neurosurgery, Brigham \& Women's Hospital, Harvard University, Boston, Massachusetts

Object. A low triiodothyronine (T3) state is highly prevalent and is associated with a poor prognosis in critically ill patients. The authors investigated, in patients undergoing brain tumor surgery, the direct association of a perioperative low T3 syndrome with clinical outcomes and also with symptoms of depression and anxiety.

Methods. Ninety consecutive patients (71\% women, median age 55 years), on admission for brain tumor surgery, were evaluated for sociodemographic and clinical characteristics. Their thyroid function profile was assessed on the morning of brain tumor surgery and on the morning after brain tumor surgery. Patients with free T3 concentrations of $3.1 \mathrm{pmol} / \mathrm{L}$ or less were considered to have low T3 syndrome. The patients were evaluated for symptoms of depression and anxiety using the Hospital Anxiety and Depression Scale (HADS) before and after surgery and for clinical outcomes using the Glasgow Outcome Scale (GOS) at discharge.

Results. After brain tumor surgery, free T3 concentrations decreased $(\mathrm{p}<0.001)$ and the proportion of patients with low T3 levels increased from $38 \%$ to $54 \%(\mathrm{p}=0.02)$. Lower preoperative (rho $=0.30, \mathrm{p}=0.004)$ and postoperative (rho $=0.33, \mathrm{p}=0.002$ ) free T3 concentrations correlated with low GOS scores at discharge. Preoperative low T3 syndrome (OR 5.49, 95\% CI 1.27-23.69, $\mathrm{p}=0.02$ ) and postoperative low T3 syndrome (OR 8.73, 95\% CI $1.49-51.21, \mathrm{p}=0.02)$ both increased risk for unfavorable clinical outcomes (GOS scores $<5)$ at discharge, after adjusting for age, sex, histological diagnosis of brain tumor, preoperative functional impairment, previous treatment for brain tumor, and depressive symptoms. Preoperative low T3 syndrome increased the risk for preoperative (HADSdepression subscale score $\geq 11$; OR $4.12,95 \%$ CI $1.16-14.58, \mathrm{p}=0.03$ ) but not postoperative depressive symptoms independently from sociodemographic and clinical factors.

Conclusions. Low T3 syndrome is a strong independent predictor of unfavorable clinical outcomes and depressive symptoms, and its diagnosis and preoperative management should be considered in patients undergoing neurosurgery for the treatment of brain tumors.

(http://thejns.org/doi/abs/10.3171/2013.1.JNS121696)

\section{$\begin{array}{llllll}\text { KEY WoRDs } & \text { brain tumor } \\ \text { clinical outcome } & \bullet \quad \text { oncology }\end{array}$}

$\mathrm{B}$ RAIN tumor is a devastating disease associated with significant functional impairment and often with a poor prognosis..$^{7,17}$ Surgery remains the most important definitive treatment modality for patients with brain tumors. Hence, early and accurate prediction of postoperative outcomes is vital for the guidance of therapy, defining discharge plans, and optimal use of health

\footnotetext{
Abbreviations used in this paper: $\mathrm{BI}=$ Barthel Index; GOS = Glasgow Outcome Scale; HADS = Hospital Anxiety and Depression Scale; HADS-A = anxiety subscale of HADS; HADS-D = depression subscale of HADS; IQR = interquartile range; TPO-Abs $=$ anti-thyroid peroxidase antibodies; TRH $=$ thyrotropin-releasing hormone; $\mathrm{TSH}$ = thyroid-stimulating hormone; T3 = triiodothyronine; $\mathrm{T} 4=$ thyroxine.
}

care resources. To date, predicting the course of disease in patients with brain tumor rests mainly on clinical disease severity indices, such as histological malignancy, extent of resection, age, and preoperative functional status. ${ }^{7,25,26}$ A new generation of genetic and molecular prognostic biomarkers related to brain tumors is emerging, but the routine use of these biomarkers remains limited by high costs, and their prognostic value warrants additional research..$^{7,20}$

Alterations of the hypothalamic-pituitary-thyroid axis are well described in critical illness and in mood disorders. ${ }^{12,46}$ Briefly, the activation of this axis involves hypothalamic release of thyrotropin-releasing hormone (TRH), which stimulates the pituitary release of thyroidstimulating hormone (TSH), which in turn activates the 
thyroid to produce and release the hormones thyroxine (T4) and triiodothyronine (T3), ${ }^{4,6}$ The primary secretory product of the thyroid gland is the less metabolically active T4, whereas the majority of the more metabolically active T3 comes from extrathyroidal conversion from T4 via deiodination in different tissues, including brain. The secretion of both TSH and TRH can be inhibited by T3 and T4 via a negative feedback loop.

A typical pattern of altered thyroid hormone metabolism in critical illness is characterized by decreased peripheral concentrations of T3 (that is, low T3 syndrome or euthyroid sick syndrome), due to reduced enzymatic activity of $5^{\prime}$ deiodinase, which is primarily responsible for T4 to T3 conversion and for T3 availability in tissues. ${ }^{12,46}$ Low T3 syndrome is highly prevalent and independently predicts a poor prognosis in hospitalized and critically ill patients. ${ }^{2,34,46}$ In surgical patients, a number of studies have documented postoperative decrease of $\mathrm{T} 3$ concentrations, and lower T3 concentrations were associated with unfavorable outcomes, suggesting that low T3 syndrome can be an important prognostic biomarker in surgical patients..$^{14,24,36,38,43,48}$ To our knowledge, however, no studies have investigated the prevalence of low T3 syndrome in the perioperative period and the direct link of low T3 syndrome to clinical outcomes in neurosurgical patients.

Depression and anxiety symptoms are considered important secondary outcomes in neurooncology, are highly prevalent in brain tumor patients, and predict poor clinical outcomes. ${ }^{3,19,28}$ Despite the well-established association of thyroid function with psychiatric symptoms in patients with psychiatric and somatic conditions, there are no studies evaluating the association of thyroid axis function with depression and anxiety in brain tumor patients. ${ }^{4,12}$ The identification of depression and anxiety biomarkers could potentially improve risk stratification and contribute to the improved care and survival of these patients.

Therefore, the aim of the present report was to evaluate, in patients undergoing brain tumor surgery, the prevalence of perioperative low T3 syndrome and the direct association of perioperative low T3 syndrome with immediate postoperative clinical outcomes as well as with symptoms of depression and anxiety.

\section{Methods}

\section{Patients}

In the period from March 2010 until June 2011, consecutive patients admitted for scheduled brain tumor surgery at the Department of Neurosurgery of the Lithuanian University of Health Sciences were eligible for this study. Patients were excluded from the study if they were younger than 18 years of age, were pregnant or nursing, had current thyroid disease, or were currently taking thyroid medication or amiodarone.

A total of 114 patients met the inclusion criteria and agreed to participate in the study. However, 24 patients (21\%) were excluded because they had elevated titers of anti-thyroid peroxidase antibodies (TPO-Abs), suggest- ing autoimmune thyroid disease. Hence, our final sample consisted of 90 brain tumor patients without frank thyroid disease (71\% women and $29 \%$ men; mean age of 55.1 \pm 13.9 years). No differences were seen in sociodemographic and clinical characteristics between patients who were excluded and those who were studied ( $p>0.08$ for all comparisons).

\section{Study Design}

The study and its consent procedures were approved by the Ethics Committee for Biomedical Research at the Lithuanian University of Health Sciences, Kaunas, Lithuania. Written informed consent was obtained from each study patient.

All study patients were enrolled on admission to the inpatient department and data were recorded for demographic characteristics, education (range from not having finished high school to graduation from college), marital status (living with partner or not living with partner), previous treatment for brain tumor (yes or no), history of psychiatric disorder (yes or no), and current psychiatric treatment (yes or no). During the same visit, patients were evaluated for functional status (Barthel Index, BI ${ }^{27}$ and for symptoms of depression and anxiety (HADS). ${ }^{49}$ The final histological diagnosis of brain tumor was established by reviewing the pathology reports, which were obtained from the medical records. Within 3 days after brain tumor surgery, patients were reevaluated using the HADS. At discharge, the clinical outcome was determined according to the score on the GOS..$^{22}$ Blood samples for the evaluation of thyroid axis hormone concentrations were drawn on 2 occasions: 1) the morning of brain tumor surgery and 2) the morning following brain tumor surgery.

\section{Functional Status}

Functional status on admission was assessed using the $\mathrm{BI}$, which is a 10 -item scale designed to evaluate daily functions of dressing, bathing, feeding, grooming, transfers from bed to chair and back, bladder and bowel control, toilet use, mobility, and climbing stairs. ${ }^{27}$ Each item is scored as $0,5,10$, or 15 , depending on the person's ability to perform the activity. The global BI score ranges from 0 to 100 points, with higher scores indicating better functional status.

\section{Evaluation for Symptoms of Depression and Anxiety}

The HADS is a 14-item self-rating instrument that consists of subscales of anxiety (HADS-A) and depression (HADS-D), which are designed to measure respective symptoms in patients with somatic conditions. ${ }^{49}$ Possible scores in both subscales range from 0 to 21 , with a higher score indicating more severe symptoms. We also evaluated the total score on the HADS (HADS-total). Scores of 11 or higher on the HADS-D and HADS-A suggest elevated depressive and anxiety symptoms, respectively. ${ }^{49}$ A Lithuanian version of the HADS is well validated and widely used on an inpatient and outpatient basis for the evaluation of anxiety and depression in patients with somatic conditions..$^{10,11}$ 


\section{Evaluation for Clinical Outcome}

Clinical outcome at discharge was evaluated by means of the 5-point GOS. ${ }^{22}$ In agreement with previous research, patients with GOS scores of 5 (good recovery) were considered as having a favorable clinical outcome and patients with GOS scores ranging from 1 (death) to 4 (moderate disability) were considered as having an unfavorable clinical outcome. ${ }^{16}$ The GOS is widely used in neurosurgical clinical practice. It is a well-validated scale with good interobserver agreement. ${ }^{47}$

\section{Thyroid Hormone Assessment}

Blood samples for thyroid axis hormone assessment were drawn from an antecubital vein about 8 AM before breakfast and within 10 minutes after patients awoke, following overnight fasting. Blood was rapidly centrifuged and serum was frozen at $-40^{\circ} \mathrm{C}$. The samples from all patients for each parameter were analyzed in a single batch. Serum concentrations of free T3, free T4, TSH, and TPO-Abs were measured by radioimmunoassay, and serum concentrations of reverse free T3 (rT3) were measured by ELISA method. The reference values for our laboratory were as follows: free T3, 3.1-6.4 pmol/L; free T4, 11.5-20.7 pmol/L; TSH, 0.27-4.2 mIU/L; and TPOAbs, less than $60 \mathrm{U} / \mathrm{ml}$. We also calculated the free T3 to free $\mathrm{T} 4$ ratio, which corresponds to peripheral free $\mathrm{T} 4$ to free $\mathrm{T} 3$ conversion, with a higher ratio corresponding to greater conversion.

On the basis of free T3 values, patients were dichotomized into 2 subgroups: 1) patients with free T3 concentrations at or below the lower concentration of the free $\mathrm{T} 3$ reference interval $(\leq 3.1 \mathrm{pmol} / \mathrm{L})$ were considered as having low T3 syndrome and 2) patients were considered to have normal free $\mathrm{T} 3$ values if their free T3 concentrations were greater than $3.2 \mathrm{pmol} / \mathrm{L}$.

\section{Statistical Analyses}

Data were analyzed with SPSS 17.0 for Windows. Data are presented as mean \pm standard deviation and as median (IQR) for continuous variables and as the number (\%) for categorical variables. We employed nonparametric statistical tests because, according to the KolmogorovSmirnov test, endocrine data and psychiatric data were not normally distributed. All tests were 2-tailed and $\mathrm{p}<$ 0.05 was considered statistically significant.

First, by using the Wilcoxon signed rank test for continuous data and the McNemar test for categorical data we evaluated the change of thyroid axis hormone concentrations and free T3 to free T4 ratio as well as symptoms of depression and anxiety before versus after brain tumor surgery.

Second, using the Spearman rank correlation, we explored the association of perioperative thyroid axis hormone concentrations and free T3 to free T4 ratios with clinical outcome at discharge. We then evaluated the association of preoperative and postoperative low T3 syndrome with clinical outcome at discharge in univariate logistic regression analyses, and the results are presented as odds ratios and $95 \%$ confidence intervals. Significant univariate associations were then adjusted in multivariate binary logistic regression model(s) (forward: LR) for age, sex, preoperative BI score, previous treatment for brain tumor, and histological diagnosis of brain tumor, because these factors were previously shown to be important prognostic factors in patients with brain tumors., ${ }^{7,25,26}$ In the final multivariate regression analyses model, the HADS-D score before surgery was included as a covariate.

Finally, the association of preoperative and postoperative thyroid axis hormone concentrations and free $\mathrm{T} 3$ to free $\mathrm{T} 4$ ratios with preoperative and postoperative HADS-D, HADS-A, and HADS-total scores were explored by using the Spearman rank correlation. The association of preoperative low T3 syndrome with elevated perioperative depressive and anxiety symptoms was evaluated by using univariate binary logistic regression analyses. We then employed multivariate binary logistic regression models and adjusted significant univariate associations (forward: LR) for age, sex, living condition, psychiatric history, current psychiatric treatment, preoperative BI score, previous treatment for brain tumor, and histological diagnosis of brain tumor.

\section{Results}

\section{Baseline Characteristics}

In 37 patients $(41 \%)$, the diagnosis was meningioma; in $17(19 \%)$, high-grade glioma; in $12(13 \%)$, pituitary adenoma; in 11 (12\%), acoustic neuroma; in 5 (6\%), low-grade glioma; and in 8 (9\%), other brain tumors, including metastatic tumors (2), hemangiopericytoma (1), germinoma (1), teratoma (1), pineal tumor (1), Rathke cleft cyst (1), and pineocytoma (1) (Table 1). Forty-two percent of patients had graduated from high school, and $76 \%$ were living with a partner. Eighteen percent of patients had previously undergone surgery for brain tumor treatment. Six percent of patients had histories of psychiatric disorders, and $8 \%$ were receiving psychotropic medication.

\section{Perioperative Thyroid Axis Function and Symptoms of Depression and Anxiety}

Thyroid hormone concentrations and ratios and symptoms of depression and anxiety before versus after brain tumor surgery are presented in Table 2. After brain tumor surgery, when compared with respective values before surgery, there was a significant decrease in free T3 concentrations, TSH concentrations, and free T3 to free $\mathrm{T} 4$ ratios ( $\mathrm{p}<0.001$ for all comparisons). There was also a significant increase in free $\mathrm{T} 4$ concentrations after brain tumor surgery $(\mathrm{p}<0.001)$. Low T3 syndrome was diagnosed in $38 \%$ of patients before surgery and in $54 \%$ of patients after surgery $(\mathrm{p}=0.02)$.

With respect to symptoms of anxiety and depression, there was a significant decrease in HADS-D scores $(\mathrm{p}=$ $0.003)$ and HADS-A scores $(\mathrm{p}<0.001)$ after brain tumor surgery (Table 2). There was also a significant decrease in the proportion of patients with elevated HADS-D scores $(16 \%$ vs $7 \%$, respectively, $\mathrm{p}=0.02)$ and a trend for a decreased proportion of patients with elevated HADS-A scores $(17 \%$ vs $8 \%$, respectively, $\mathrm{p}=0.057)$ (Fig. 1$)$. 
TABLE 1: Baseline sociodemographic, clinical, and psychiatric data for 90 patients*

\begin{tabular}{|c|c|}
\hline Characteristic & Value \\
\hline \multicolumn{2}{|l|}{ sex } \\
\hline male & $26(29)$ \\
\hline female & $64(71)$ \\
\hline \multicolumn{2}{|l|}{ age in yrs } \\
\hline mean & $55.1 \pm 13.9$ \\
\hline median & 55 \\
\hline IQR & $46-66$ \\
\hline no. of pts $(\%)$ w/ age $>50$ yrs & $62(69)$ \\
\hline \multicolumn{2}{|l|}{ education } \\
\hline not graduated from high school & $5(6)$ \\
\hline graduated from high school & $38(42)$ \\
\hline some college & $24(27)$ \\
\hline graduated from college & $23(25)$ \\
\hline \multicolumn{2}{|l|}{ marital status } \\
\hline living w/ partner & $68(76)$ \\
\hline not living w/ partner & $22(24)$ \\
\hline \multicolumn{2}{|l|}{ histological diagnosis of brain tumor } \\
\hline high-grade glioma & $17(19)$ \\
\hline low-grade glioma & $5(6)$ \\
\hline meningioma & $37(41)$ \\
\hline pituitary adenoma & $12(13)$ \\
\hline acoustic neuroma & $11(12)$ \\
\hline other & $8(9)$ \\
\hline \multicolumn{2}{|l|}{ previous brain tumor treatment } \\
\hline yes & $16(18)$ \\
\hline no & $74(82)$ \\
\hline \multicolumn{2}{|l|}{ Barthel Index } \\
\hline mean score & $94.9 \pm 13.6$ \\
\hline median score & 100 \\
\hline IQR & $100-100$ \\
\hline \multicolumn{2}{|l|}{ history of psychiatric disorder } \\
\hline yes & $5(6)$ \\
\hline no & $85(94)$ \\
\hline \multicolumn{2}{|l|}{ current psychiatric treatment } \\
\hline yes & $7(8)$ \\
\hline no & $83(92)$ \\
\hline
\end{tabular}

* Unless otherwise indicated, values represent numbers of patients (pts) (\%). Means are presented with SD.

\section{Association of Thyroid Axis Function With Clinical Outcome}

At discharge, the median GOS score was 5, and 18 patients $(20 \%)$ had GOS scores of 4 or lower, indicative of unfavorable clinical outcomes (including postoperative death in 2 cases).

Correlation analyses revealed that lower preoperative free T3 concentration ( $\mathrm{Tho}=0.30, \mathrm{p}=0.004$ ), lower postoperative free $\mathrm{T} 3$ concentration ( $\mathrm{rho}=0.33, \mathrm{p}=0.002$ ), and lower preoperative free $\mathrm{T} 3$ to free $\mathrm{T} 4$ ratio (that is, lower T4 to $\mathrm{T} 3$ conversion; rho $=0.23, \mathrm{p}=0.03$ ) were associated with lower GOS scores (that is, unfavorable outcome) at discharge (Table 3 ). Other perioperative thyroid hormone concentrations did not correlate significantly with GOS scores at discharge.

In univariate binary regression analyses, patients with low T3 syndrome before surgery (OR 4.46, 95\% CI $1.48-13.40, \mathrm{p}=0.008$ ) and after surgery (OR 5.44, 95\% CI 1.45-20.45, p = 0.012; Table 4) were at increased risk for unfavorable clinical outcomes at discharge relative to patients with normal free T3 concentrations. After adjusting for age, sex, preoperative functional impairment, previous treatment for brain tumor, and histological diagnosis of brain tumor, preoperative low T3 syndrome (OR 5.09, 95\% CI 1.38-18.78, $\mathrm{p}=0.01$ ) and postoperative low T3 syndrome (OR 5.35, 95\% CI 1.20-23.90, p = 0.03) were associated with an independently increased risk for unfavorable clinical outcome at discharge. When the preoperative HADS-D score was included as an additional covariate in the multivariate binary regression analyses model, preoperative low T3 syndrome (OR 5.49, 95\% CI $1.27-23.69, \mathrm{p}=0.02$ ) and postoperative low $\mathrm{T} 3$ syndrome (OR 8.73, 95\% CI 1.49-51.21, $\mathrm{p}=0.02$ ) remained independently associated with increased risk of an unfavorable clinical outcome at discharge.

\section{Association of Thyroid Axis Function With Symptoms of Depression and Anxiety}

Lower preoperative free T3 concentration was associated with greater preoperative HADS-D (rho $=-0.22$, $\mathrm{p}=0.04)$, HADS-A (rho $=-0.25, \mathrm{p}=0.02)$, and HADStotal (rho $=-0.31, \mathrm{p}=0.003$ ) scores, and with greater postoperative HADS-D (rho $=-0.22, \mathrm{p}=0.04$ ) and HADS-total (rho $=-0.23, \mathrm{p}=0.03$ ) scores. Also, lower preoperative free $\mathrm{T} 3$ to free $\mathrm{T} 4$ ratio (that is, lower $\mathrm{T} 4$ to T3 conversion) was associated with greater preoperative HADS-D (rho $=-0.25, \mathrm{p}=0.02)$ and HADS-total (rho = $-0.24, p=0.03$ ) scores (Table 5). Postoperative thyroid axis hormone concentrations and free T3 to free T4 ratio were not associated with postoperative HADS-D, HADSA, or HADS-total scores ( $\mathrm{p} \geq 0.12$ for all comparisons; data not shown).

In univariate binary logistic regression analyses, preoperative low T3 syndrome was associated with increased risk for elevated preoperative depressive symptoms (OR $3.60,95 \%$ CI 1.09-11.88, $\mathrm{p}=0.035)$, but not preoperative anxiety symptoms (Table 6). After adjusting for age, sex, living conditions, psychiatric history, current psychiatric treatment, functional impairment, previous treatment for brain tumor, and histological diagnosis of brain tumor, the preoperative low T3 syndrome remained an independent predictor of elevated preoperative depressive symptoms (OR 4.12, 95\% CI 1.16-14.58, $\mathrm{p}=0.03$ ). Preoperative low T3 syndrome was not associated with increased risk for postoperative depressive and anxiety symptoms.

\section{Discussion}

The main findings of the present study were that perioperative low T3 syndrome was highly prevalent in pa- 
TABLE 2: Thyroid axis function and scores on the HADS before versus after brain tumor surgery

\begin{tabular}{|c|c|c|c|c|c|c|c|}
\hline Variable & Preop* & Postop* & $\mathrm{Z} / \chi^{2}$ & $\begin{array}{c}p \\
\text { Value† }\end{array}$ & Increasef & Decreasef & $\begin{array}{c}\text { No } \\
\text { Changef }\end{array}$ \\
\hline \multicolumn{8}{|l|}{ thyroid axis hormone concentrations } \\
\hline free T3, pmol/L & $3.5(3.0-4.1)$ & $3.2(2.7-3.6)$ & -4.81 & $<0.001$ & 24 & 64 & 0 \\
\hline free $T 4$, pmol/L & $12.6(11.3-14.7)$ & $17.7(15.4-20.9)$ & -8.16 & $<0.001$ & 86 & 4 & 0 \\
\hline reverse T3, pg/ml & $866(620-1074)$ & $884(689-1168)$ & -0.98 & 0.33 & 52 & 38 & 0 \\
\hline TSH, mIU/L & $0.70(0.36-1.40)$ & $0.32(0.20-0.57)$ & -5.99 & $<0.001$ & 16 & 73 & 0 \\
\hline free T3/free T4 ratios & $0.27(0.22-0.32)$ & $0.17(0.15-0.20)$ & -8.16 & $<0.001$ & 2 & 86 & 0 \\
\hline no. of pts (\%) w/ low T3 syndrome§ & $34(38)$ & $49(54)$ & 5.28 & 0.02 & & & \\
\hline \multicolumn{8}{|l|}{ HADS scores } \\
\hline HADS-D & $4(1-7)$ & $2(1-6)$ & -3.00 & 0.003 & 21 & 37 & 23 \\
\hline HADS-A & $5(2-9)$ & $4(1-7)$ & -4.43 & $<0.001$ & 18 & 55 & 8 \\
\hline
\end{tabular}

* Unless otherwise indicated, preoperative and postoperative values represent medians (IQR).

$\dagger$ Bold type indicates statistical significance $(p<0.05)$.

$\ddagger$ Values represent numbers of patients.

$\S$ Low T3 syndrome is defined by a free T3 (triiodothyronine) concentration $\leq 3.1 \mathrm{pmol} / \mathrm{L}$.

tients undergoing brain tumor surgery and was associated with increased risk for unfavorable clinical outcomes at discharge, and preoperative low T3 syndrome was associated with increased risk for preoperative depressive symptoms.

To the best of our knowledge, this is the first study to examine perioperative thyroid axis function in patients undergoing brain tumor surgery. The preoperative prevalence rate of low T3 syndrome $(38 \%)$ in brain tumor patients corresponds to low T3 syndrome prevalence rates previously reported in hospitalized cardiac patients $(30 \%)^{21}$ and in acutely ill elderly patients $(32 \%){ }^{41}$ As expected, median free T3 concentrations decreased and the prevalence of low T3 syndrome (54\%) increased within 24 hours after brain tumor surgery. A number of previous studies have documented decreased T3 concentrations within a few hours after major surgery, returning to preoperative levels within a week after surgery. ${ }^{14,43,48}$ Comparable prevalence rates of postoperative low $\mathrm{T} 3$ syndrome were previously reported after kidney transplantation (52\%). ${ }^{40}$ It is widely accepted that decreased peripheral $5^{\prime}$ monodeiodinase activity is the chief pathophysiological mechanism underlying the development of low T3 syndrome in critical illness. ${ }^{44,46}$ Indeed, we found that the free T3 to free T4 ratio (an index of peripheral $5^{\prime}$ monodeiodinase activity) was significantly decreased after surgery when compared with preoperative levels. A number of factors can interfere with thyroid hormone metabolism; these factors include but are not limited to endogenous stress hormones and proinflammatory cytokines (not evaluated), concentrations of which are expected to elevate after surgery. ${ }^{1,42,44,46}$ In line with our results, a decrease in TSH concentration was previously described in critically ill patients and in patients who had undergone surgical procedures. ${ }^{1,23,46}$ Decreased TSH secretion results in decreased thyroidal T3 and T4 production and contributes to the development of low $\mathrm{T} 3$ syndrome. It was shown that a prolonged low T3 syndrome can be associated with decreased hypothalamic
TRH synthesis. ${ }^{18}$ Also, postoperative TSH and/or TRH secretion can be suppressed by elevated free T4 concentrations, by postoperative increases in concentrations of proinflammatory cytokines and endogenous cortisol, and by caloric restriction. ${ }^{1,42,46}$ The clinical significance of T4 in critical illness remains less clear, and findings regarding postoperative changes in T4 concentrations have been inconsistent, given that it was reported that T4 concentrations may increase, ${ }^{15}$ decrease, ${ }^{48}$ or remain unchanged ${ }^{43}$ after surgery. The postoperative increase in free $\mathrm{T} 4$ concentrations found in our study could be due to 1) suppressed conversion of T3 from T4 leading to a build-up of $\mathrm{T} 4 ; 2$ ) impaired entry of free T4 into cells in patients with low T3 syndrome; ${ }^{37}$ and 3) increased release of T4 from thyroid gland stores together with impaired function of thyroid-binding globulin previously described in relation to certain anesthetics, such as isoflurane and enflurane, that are widely used in neurosurgery. ${ }^{8,15,29}$

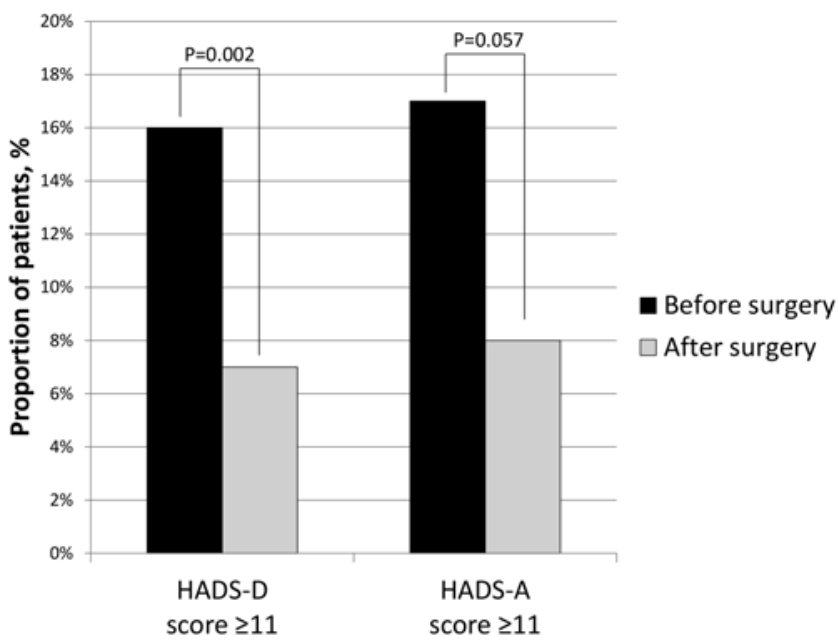

FIG. 1. Proportion of patients with scores of 11 or higher on the depression and anxiety subscales of the HADS before and after brain tumor surgery. 
TABLE 3: Association of perioperative thyroid axis function with GOS at discharge*

\begin{tabular}{ccc}
\hline Hormone Concentration or Ratio & Spearman rho & p Value \\
\hline free T3 in pmol/L & & \\
preop & 0.30 & 0.004 \\
postop & 0.33 & 0.002 \\
free T4 in pmol/L & & \\
preop & 0.04 & 0.70 \\
postop & 0.07 & 0.49 \\
reverse T3 in pg/ml & & \\
preop & 0.13 & 0.21 \\
postop & 0.08 & 0.44 \\
TSH in mlU/L & & \\
preop & 0.02 & 0.85 \\
postop & -0.14 & 0.20 \\
free T3/free T4 ratio & & \\
preop & 0.23 & 0.03 \\
postop & 0.20 & 0.06 \\
\hline
\end{tabular}

* Greater scores indicate better clinical outcome. Bold type indicates statistical significance $(p<0.05)$.

Most importantly, we found that perioperative low T3 syndrome was independently associated with a 5- to 8 -fold increased risk for unfavorable clinical outcomes at discharge, even after adjusting for age, sex, preoperative functional impairment, previous treatment for brain tumor, histological diagnosis of brain tumor, and preoperative depressive symptoms. It should be noted that inclusion of brain tumor type, including pituitary adenomas, to the models did not change the association between thyroid hormone concentrations and outcomes of brain surgery. In correlation analyses, a lower perioperative free T3 concentration was associated with poor outcomes, suggesting a dose-response relationship between low free T3 concentrations (even within a normal range) and poor clinical outcome. Our results regarding the independent prognostic role of low T3 syndrome correspond to results of previous studies showing that low T3 syndrome was an independent predictor of mortality in different populations of patients, including cardiac patients ${ }^{21}$ stroke patients, ${ }^{2}$ and hospitalized elderly patients. ${ }^{41} \mathrm{~A}$ dose-response relationship between low T3 concentrations and poor outcomes was previously described in hospitalized patients ${ }^{46}$ and in general surgery patients. ${ }^{43}$ Triiodothyronine is essential for proper functioning of the brain and cardiovascular system, and results from preclinical and clinical studies suggest that thyroid hormones can promote plasticity and regeneration in the CNS. ${ }^{33,39}$ Further studies should explore mechanisms underlying the negative impact of low T3 syndrome. Nevertheless, clinicians should keep in mind that low T3 syndrome is highly prevalent and independently predicts poor prognosis in brain tumor patients. Patients undergoing surgery for treatment of brain tumors who have perioperative free $\mathrm{T} 3$ concentrations at or below the normal range should be considered at high risk for poor outcomes.

An important benefit of low T3 syndrome as a prognostic biomarker is that it can be easily treated by using thyroid hormone replacement therapy. However, the need for thyroid hormone replacement therapy in patients with low T3 syndrome remains controversial. ${ }^{44,46}$ For example, in patients with heart failure ${ }^{32}$ or in patients undergoing heart surgery, ${ }^{24,35}$ thyroid hormone replacement therapy was safe and improved hemodynamic function; however, the benefit with respect to mortality remains less clear. Others have reported that thyroid hormone replacement therapy did not improve clinical outcomes in intensive care unit patients ${ }^{9}$ and in patients with burn injuries. ${ }^{5}$ Nonetheless, thyroid hormone replacement therapy in low T3 patients undergoing brain surgery is an interesting avenue for future research, because thyroid hormones cross the blood-brain barrier and are critical for proper development, function, and regeneration of the CNS.,12,46

In our patient group, depression and anxiety were prevalent before surgery and improved after surgery. According to the literature, the prevalence of depression in brain tumor patients ranges from $5 \%$ for active clinical depression $^{19}$ to $40 \%$ for elevated depressive symptoms. ${ }^{3}$ Lower prevalence rates of depressive and anxiety symptoms in our study can be explained by our having considered patients to have elevated depressive and/or anxiety symptoms if they had moderate to severe levels of respective symptoms if their HADS subscale scores 11 or greater. Gathinji and colleagues ${ }^{19}$ reported that preoperative clinical depression independently predicted worse survival of patients with brain tumor, underscoring the fact that depression is an important risk factor that should be promptly diagnosed and treated. In the present study, low T3 syndrome predicted a 4-fold increased risk for el-

TABLE 4: Association of preoperative and postoperative low T3 syndrome with unfavorable clinical outcome at discharge

\begin{tabular}{|c|c|c|c|c|c|c|c|c|c|}
\hline \multirow[b]{2}{*}{ Variable } & \multicolumn{3}{|c|}{ Univariate Association } & \multicolumn{3}{|c|}{ Multivariate Association* } & \multicolumn{3}{|c|}{ Multivariate Association† } \\
\hline & OR & $95 \% \mathrm{Cl}$ & p Valuef & OR & $95 \% \mathrm{Cl}$ & p Valuef & OR & $95 \% \mathrm{Cl}$ & p Valuef \\
\hline \multicolumn{10}{|c|}{ predictor: discharge GOS score $<5$} \\
\hline postop low T3 syndrome & 5.44 & $1.45-20.45$ & 0.012 & 5.35 & $1.20-23.90$ & 0.03 & 8.73 & $1.49-51.21$ & 0.02 \\
\hline
\end{tabular}

\footnotetext{
* Adjusted for age, sex, preoperative BI score, previous brain tumor treatment, and histological diagnosis of brain tumor (forward: LR).

$\dagger$ Adjusted for age, sex, preoperative BI score, previous brain tumor treatment, histological diagnosis of brain tumor, and HADS-D score before surgery (forward: LR).

$\ddagger$ Bold type indicates statistical significance $(p<0.05)$.
} 
Low T3 syndrome in brain tumor surgery

TABLE 5: Association of preoperative thyroid axis function with perioperative symptoms of depression and anxiety*

\begin{tabular}{|c|c|c|c|c|c|c|}
\hline \multirow{2}{*}{$\begin{array}{c}\text { Preop Hormone } \\
\text { Concentration or Ratio }\end{array}$} & \multicolumn{3}{|c|}{ Preop HADS Score $†$} & \multicolumn{3}{|c|}{ Postop HADS Score $†$} \\
\hline & Depression & Anxiety & Total & Depression & Anxiety & Total \\
\hline free T3 in $\mathrm{pmol} / \mathrm{L}$ & $-0.22(0.04)$ & $-0.25(0.02)$ & $-0.31(0.003)$ & $-0.22(0.04)$ & $-0.14(0.18)$ & $-0.23(0.03)$ \\
\hline free $\mathrm{T} 4$ in $\mathrm{pmol} / \mathrm{L}$ & $0.01(0.95)$ & $-0.21(0.055)$ & $-0.10(0.95)$ & $-0.04(0.74)$ & $-0.17(0.11)$ & $-0.08(0.46)$ \\
\hline reverse $\mathrm{T} 3, \mathrm{pg} / \mathrm{mL}$ & $0.17(0.11)$ & $-0.04(0.72)$ & $0.06(0.58)$ & $0.08(0.45)$ & $0.12(0.27)$ & $0.12(0.28)$ \\
\hline TSH in mIU/L & $-0.01(0.90)$ & $0.11(0.29)$ & $0.02(0.85)$ & $0.06(0.60)$ & $0.15(0.17)$ & $0.10(0.35)$ \\
\hline free T3/free T4 ratio & $-0.25(0.02)$ & $-0.13(0.29)$ & $-0.24(0.03)$ & $-0.19(0.07)$ & $-0.04(0.74)$ & $-0.17(0.11)$ \\
\hline
\end{tabular}

* Values represent Spearman rho with $p$ value in parentheses. Bold type indicates statistical significance $(p<0.05)$.

$\dagger$ Greater scores indicate more symptoms.

evated preoperative depressive symptoms, independently from age, sex, living condition, psychiatric histories and treatment, functional impairment, previous treatment for brain tumor, and histological diagnosis of brain tumor, including pituitary adenomas. In univariate analyses, lower T3 to T4 ratios were associated with greater depressive symptoms, suggesting that decreased activity of $5^{\prime}$ deiodinase can be related to depressive symptom severity in brain tumor patients. The association of low T3 concentrations with elevated depressive symptoms has been previously reported in medical patients, ${ }^{13}$ and it was suggested that elevated hypothalamic-pituitary-adrenal axis activity in depressed patients can suppress $5^{\prime}$ deiodination leading to decreased T3 concentrations. ${ }^{31}$ In addition, it is well established that depression is prevalent in hypothyroid patients. ${ }^{4,12}$ However, due to the design of our study we could not establish the directional association between thyroid function and depression. Nevertheless, our data suggest that decreased free T3 concentrations can be an important biomarker for the development of depressive symptoms in brain tumor patients; hence, free $\mathrm{T} 3$ evaluation should to be considered if patients in this population are depressed.

It should be recalled that we evaluated peripheral concentrations of thyroid hormones. Thyroid hormones undergo significant metabolism, a variety of transporters are responsible for thyroid hormone tissue concentrations, and thyroid hormone receptors have a complex mode of action. $.12,46$ The activity and expression of proteins involved in metabolism, transfer, and binding of thyroid hormones can differ and/or can be differentially modified in response to low T3 syndrome as a function of certain genetic polymorphisms. ${ }^{30,45,46}$ These genetic polymorphisms can consequentially result in different clinical and neuropsychiatric outcomes in patients affected by low T3 syndrome. Thus, future studies should explore outcomes of brain tumor patients in relation to genetic polymorphisms of proteins involved in metabolism, transportation, and binding of thyroid hormones. This knowledge could enable more precise identification of high-risk patients based on inherent genetic polymorphisms.

Our study has limitations. First, because of the relatively small sample size, our results should be considered preliminary. In addition, it should be noted that our cohort was heterogeneous in terms of clinical characteristics, such as brain tumor biology and previous treatments for brain tumor; therefore, further studies should explore the impact of low T3 syndrome in more discrete subgroups of brain tumor patients. A larger study evaluating the association of outcomes with low T3 syndrome and with genetic polymorphisms of proteins involved in metabolism and transfer of thyroid hormones is under

TABLE 6: Association of preoperative low T3 syndrome with preoperative and postoperative depressive and anxiety symptoms

\begin{tabular}{|c|c|c|c|c|c|c|}
\hline \multirow[b]{2}{*}{ Variable } & \multicolumn{3}{|c|}{ Univariate Association } & \multicolumn{3}{|c|}{ Multivariate Association* } \\
\hline & OR & $95 \% \mathrm{Cl}$ & p Value & OR & $95 \% \mathrm{Cl}$ & $\mathrm{p}$ Value \\
\hline \multicolumn{7}{|c|}{ predictor: preop depression† } \\
\hline preop low T3 syndrome & 3.60 & $1.09-11.88$ & 0.035 & 4.12 & $1.16-14.58$ & 0.03 \\
\hline \multicolumn{7}{|l|}{ predictor: preop anxiety } \\
\hline preop low T3 syndrome & 2.11 & $0.39-6.47$ & 0.19 & - & - & - \\
\hline \multicolumn{7}{|c|}{ predictor: postop depression† } \\
\hline preop low T3 syndrome & 1.73 & $0.33-9.14$ & 0.52 & - & - & - \\
\hline \multicolumn{7}{|l|}{ predictor: postop anxiety $\ddagger$} \\
\hline preop low T3 syndrome & 1.28 & $0.27-6.09$ & 0.76 & - & - & - \\
\hline
\end{tabular}

* Adjusted for age, sex, living condition, psychiatric history, current psychiatric treatment, preoperative BI score, previous brain tumor treatment, and histological diagnosis of brain tumor (forward: LR). Bold type indicates statistical significance $(p<0.05)$.

$\dagger$ Defined as HADS-D score $\geq 11$.

$\ddagger$ Defined as HADS-A score $\geq 11$. 
way. Also, levels of total (free + bound) thyroid hormone concentrations were not evaluated in our study. However, total thyroid hormone concentration is expected to be a less reliable marker of thyroid status in severe illness because thyroid hormone binding proteins are acute phase reactants.

A strength of this study is that we excluded patients with known thyroid disease, patients taking thyroid medication, and patients in whom autoimmune thyroid diseases was suspected, thus avoiding endocrine bias. Moreover, reliable and widely used instruments were used to measure disease severity, mental symptoms, and outcome, thus fortifying the reliability of our results. However, future studies should investigate the association of low T3 syndrome with more discrete clinical and neuropsychological outcomes.

\section{Conclusions}

In conclusion, perioperative low $\mathrm{T} 3$ syndrome is highly prevalent in patients undergoing brain tumor surgery and is independently associated with increased risk for unfavorable clinical outcomes and depressive symptoms. Free T3 concentration should be considered as a prognostic biomarker in neurosurgical brain tumor patients.

\section{Disclosure}

This research was funded by a grant (MIP-10315) from the Research Council of Lithuania. The authors report no conflict of interest concerning the materials or methods used in this study or the findings specified in this paper.

Author contributions to the study and manuscript preparation include the following. Conception and design: A Bunevicius, Deltuva, A Tamasauskas, R Bunevicius. Acquisition of data: S Tamasauskas. Analysis and interpretation of data: A Bunevicius, Laws, R Bunevicius. Drafting the article: A Bunevicius, Deltuva. Critically revising the article: all authors. Reviewed submitted version of manuscript: all authors. Approved the final version of the manuscript on behalf of all authors: A Bunevicius. Statistical analysis: A Bunevicius, R Bunevicius. Administrative/technical/material support: Deltuva, A Tamasauskas, R Bunevicius. Study supervision: S Tamasauskas, A Tamasauskas, R Bunevicius.

\section{Acknowledgment}

The authors thank Ms. Lina Piliciauskiene for help collecting blood samples.

\section{References}

1. Adami HO, Johansson H, Thorén L, Wide L, Akerström G: Serum levels of TSH, T3, rT3, T4 and T3-resin uptake in surgical trauma. Acta Endocrinol (Copenh) 88:482-489, 1978

2. Alevizaki M, Synetou M, Xynos K, Pappa T, Vemmos KN: Low triiodothyronine: a strong predictor of outcome in acute stroke patients. Eur J Clin Invest 37:651-657, 2007

3. Arnold SD, Forman LM, Brigidi BD, Carter KE, Schweitzer HA, Quinn HE, et al: Evaluation and characterization of generalized anxiety and depression in patients with primary brain tumors. Neuro Oncol 10:171-181, 2008

4. Bauer M, Goetz T, Glenn T, Whybrow PC: The thyroid-brain interaction in thyroid disorders and mood disorders. J Neuroendocrinol 20:1101-1114, 2008
5. Becker RA, Vaughan GM, Ziegler MG, Seraile LG, Goldfarb IW, Mansour EH, et al: Hypermetabolic low triiodothyronine syndrome of burn injury. Crit Care Med 10:870-875, 1982

6. Bianco AC, Larsen PR: Intracellular pathways of iodothyronine metabolism, in Braverman LE, Utiger RD (eds): Werner and Ingbar's The Thyroid: A Fundamental and Clinical Text, ed 9. Philadelphia: Lippincott \& Wilkins, 2005, pp 109-134

7. Bondy ML, Scheurer ME, Malmer B, Barnholtz-Sloan JS, Davis FG, Il'yasova D, et al: Brain tumor epidemiology: consensus from the Brain Tumor Epidemiology Consortium. Cancer 113 (7 Suppl):1953-1968, 2008

8. Börner U, Klimek M, Schoengen H, Lynch J, Peschau C, Schicha $\mathrm{H}$ : The influence of various anesthetics on the release and metabolism of thyroid hormones: results of two clinical studies. Anesth Analg 81:612-618, 1995

9. Brent GA, Hershman JM: Thyroxine therapy in patients with severe nonthyroidal illnesses and low serum thyroxine concentration. J Clin Endocrinol Metab 63:1-8, 1986

10. Bunevicius A, Peceliuniene J, Mickuviene N, Valius L, Bunevicius R: Screening for depression and anxiety disorders in primary care patients. Depress Anxiety 24:455-460, 2007

11. Bunevicius A, Staniute M, Brozaitiene J, Bunevicius R: Diagnostic accuracy of self-rating scales for screening of depression in coronary artery disease patients. J Psychosom Res 72:22-25, 2012

12. Bunevicius R, Prange AJ Jr: Thyroid disease and mental disorders: cause and effect or only comorbidity? Curr Opin Psychiatry 23:363-368, 2010

13. Bunevicius R, Varoneckas G, Prange AJ Jr, Hinderliter AL, Gintauskiene V, Girdler SS: Depression and thyroid axis function in coronary artery disease: impact of cardiac impairment and gender. Clin Cardiol 29:170-174, 2006

14. Burr WA, Black EG, Griffiths RS, Hoffenberg R: Serum triiodothyronine and reverse triiodothyronine concentrations after surgical operation. Lancet 2:1277-1279, 1975

15. Chikenji T, Mizutani M, Kitsukawa Y: Anaesthesia, not surgical stress, induces increases in serum concentrations of reverse triiodothyronine and thyroxine during surgery. Exp Clin Endocrinol 95:217-223, 1990

16. de Gans J, van de Beek D : Dexamethasone in adults with bacterial meningitis. N Engl J Med 347:1549-1556, 2002

17. DeAngelis LM: Brain tumors. N Engl J Med 344:114-123, 2001

18. Fliers E, Guldenaar SE, Wiersinga WM, Swaab DF: Decreased hypothalamic thyrotropin-releasing hormone gene expression in patients with nonthyroidal illness. J Clin Endocrinol Metab 82:4032-4036, 1997

19. Gathinji M, McGirt MJ, Attenello FJ, Chaichana KL, Than $\mathrm{K}$, Olivi A, et al: Association of preoperative depression and survival after resection of malignant brain astrocytoma. Surg Neurol 71:299-303, 2009

20. Huttner A: Overview of primary brain tumors: pathologic classification, epidemiology, molecular biology, and prognostic markers. Hematol Oncol Clin North Am 26:715-732, 2012

21. Iervasi G, Pingitore A, Landi P, Raciti M, Ripoli A, Scarlattini M, et al: Low-T3 syndrome: a strong prognostic predictor of death in patients with heart disease. Circulation 107:708713, 2003

22. Jennett B, Bond M: Assessment of outcome after severe brain damage: a practical scale. Lancet 1:480-484, 1975

23. Juma AH, Ardawi MS, Baksh TM, Serafi AA: Alterations in thyroid hormones, cortisol, and catecholamine concentration in patients after orthopedic surgery. J Surg Res 50:129-134, 1991

24. Klemperer JD, Klein I, Gomez M, Helm RE, Ojamaa K, Thomas SJ, et al: Thyroid hormone treatment after coronaryartery bypass surgery. N Engl J Med 333:1522-1527, 1995

25. Lamborn KR, Chang SM, Prados MD: Prognostic factors for 
survival of patients with glioblastoma: recursive partitioning analysis. Neuro Oncol 6:227-235, 2004

26. Laws ER, Parney IF, Huang W, Anderson F, Morris AM, Asher A, et al: Survival following surgery and prognostic factors for recently diagnosed malignant glioma: data from the Glioma Outcomes Project. J Neurosurg 99:467-473, 2003

27. Mahoney FI, Barthel DW: Functional evaluation: the Barthel Index. Md State Med J 14:61-65, 1965

28. Mainio A, Hakko H, Timonen M, Niemelä A, Koivukangas J, Räsänen P: Depression in relation to survival among neurosurgical patients with a primary brain tumor: a 5-year followup study. Neurosurgery 56:1234-1242, 2005

29. Marana E, Colicci S, Meo F, Marana R, Proietti R: Neuroendocrine stress response in gynecological laparoscopy: TIVA with propofol versus sevoflurane anesthesia. J Clin Anesth 22:250-255, 2010

30. Mebis L, Paletta D, Debaveye Y, Ellger B, Langouche L, D'Hoore A, et al: Expression of thyroid hormone transporters during critical illness. Eur J Endocrinol 161:243-250, 2009

31. Nemeroff CB: Clinical significance of psychoneuroendocrinology in psychiatry: focus on the thyroid and adrenal. J Clin Psychiatry 50 Suppl:13-22, 1989

32. Pingitore A, Galli E, Barison A, Iervasi A, Scarlattini M, Nucci D, et al: Acute effects of triiodothyronine (T3) replacement therapy in patients with chronic heart failure and lowT3 syndrome: a randomized, placebo-controlled study. J Clin Endocrinol Metab 93:1351-1358, 2008

33. Pitts LH, Ross A, Chase GA, Faden AI: Treatment with thyrotropin-releasing hormone $(\mathrm{TRH})$ in patients with traumatic spinal cord injuries. J Neurotrauma 12:235-243, 1995

34. Plikat K, Langgartner J, Buettner R, Bollheimer LC, Woenckhaus U, Schölmerich J, et al: Frequency and outcome of patients with nonthyroidal illness syndrome in a medical intensive care unit. Metabolism 56:239-244, 2007

35. Ranasinghe AM, Bonser RS: Thyroid hormone in cardiac surgery. Vascul Pharmacol 52:131-137, 2010

36. Reinhardt W, Misch C, Jockenhövel F, Wu SY, Chopra I, Philipp T, et al: Triiodothyronine (T3) reflects renal graft function after renal transplantation. Clin Endocrinol (Oxf) 46:563-569, 1997

37. Rodriguez-Perez A, Palos-Paz F, Kaptein E, Visser TJ, Dominguez-Gerpe L, Alvarez-Escudero J, et al: Identification of molecular mechanisms related to nonthyroidal illness syndrome in skeletal muscle and adipose tissue from patients with septic shock. Clin Endocrinol (Oxf) 68:821-827, 2008

38. Schulte C, Reinhardt W, Beelen D, Mann K, Schaefer U: Low T3-syndrome and nutritional status as prognostic factors in patients undergoing bone marrow transplantation. Bone Marrow Transplant 22:1171-1178, 1998

39. Sui L, Ren WW, Li BM: Administration of thyroid hormone increases reelin and brain-derived neurotrophic factor expression in rat hippocampus in vivo. Brain Res 1313:9-24, 2010
40. Tauchmanovà L, Carrano R, Musella T, Orio F, Sabbatini M, Lombardi G, et al: Thyroid function and morphology after a successful kidney transplantation. J Endocrinol Invest 29: 625-632, 2006

41. Tognini S, Marchini F, Dardano A, Polini A, Ferdeghini M, Castiglioni M, et al: Non-thyroidal illness syndrome and short-term survival in a hospitalised older population. Age Ageing 39:46-50, 2010

42. Torpy DJ, Tsigos C, Lotsikas AJ, Defensor R, Chrousos GP, Papanicolaou DA: Acute and delayed effects of a single-dose injection of interleukin- 6 on thyroid function in healthy humans. Metabolism 47:1289-1293, 1998

43. Tsuchiya A, Goretzki PE, Gramse M, Joseph K, Wahl RA: Low T3 syndrome in patients following major surgery. Jpn J Surg 15:285-290, 1985

44. Utiger RD: Altered thyroid function in nonthyroidal illness and surgery. To treat or not to treat? N Engl J Med 333:15621563,1995

45. van der Deure WM, Appelhof BC, Peeters RP, Wiersinga WM, Wekking EM, Huyser J, et al: Polymorphisms in the brain-specific thyroid hormone transporter OATP1C1 are associated with fatigue and depression in hypothyroid patients. Clin Endocrinol (Oxf) 69:804-811, 2008

46. Warner MH, Beckett GJ: Mechanisms behind the non-thyroidal illness syndrome: an update. J Endocrinol 205:1-13, 2010

47. Wilson JTL, Pettigrew LEI, Teasdale GM: Structured interviews for the Glasgow Outcome Scale and the extended Glasgow Outcome Scale: guidelines for their use. J Neurotrauma 15:573-585, 1998

48. Zaloga GP, Chernow B, Smallridge RC, Zajtchuk R, HallBoyer K, Hargraves R, et al: A longitudinal evaluation of thyroid function in critically ill surgical patients. Ann Surg 201: 456-464, 1985

49. Zigmond AS, Snaith RP: The hospital anxiety and depression scale. Acta Psychiatr Scand 67:361-370, 1983

Manuscript submitted August 30, 2012.

Accepted January 29, 2013.

Portions of this work were previously presented in poster form at the 10th World Congress of Biological Psychiatry, Prague, Czech Republic, May 29-June 2, 2011; 24th Congress of the European College of Neuropsychopharmacology, Paris, France, September 1-5, 2011; and Annual Meeting of the Congress of Neurological Surgeons, Chicago, Illinois, October 6-10, 2012.

Please include this information when citing this paper: published online March 12, 2013; DOI: 10.3171/2013.1.JNS121696.

Address correspondence to: Adomas Bunevicius, M.D., Department of Neurology, University of North Carolina at Chapel Hill, Physicians Office Building, 170 Manning Drive, Chapel Hill, North Carolina 27599. email: a.bunevicius@yahoo.com. 